\title{
Fungicidal versus Fungistatic activity of five Iranian essences against fluconazole resistant $C$ andida species
}

\author{
Donya Nikaein ${ }^{1,2^{*}}$, Aghil Sharifzadeh ${ }^{1,2}$, Ali Reza Khosravi ${ }^{1,2}$ \\ ${ }^{1}$ Mycology Research Center, Faculty of Veterinary Medicine, University of Tehran, Tehran, Iran \\ ${ }^{2}$ Department of Microbiology and Immunology, Faculty of Veterinary Medicine, University of Tehran, Tehran, Iran
}

\section{AR T I C L E I N F O}

\section{Article Type:}

Original Article

\section{Article History:}

Received: 19 March 2018

Accepted: 14 August 2018

\section{Keywords:}

Fungistatic activity

Fungicidal activity

Candida spp

Essential oils

Antifungal agents

\begin{abstract}
A B S T R A C T
Introduction: Antifungal resistant is one of the causes of high mortality rates during invasive candidiasis. Since development of new antifungal agents is limited, researchers have focused on natural products including essential oils (EOs) with antifungal properties. In immunocompromised patients fungicidal activity is of benefit. This study was designed to evaluate chemical composition and fungicidal/fungistatic activities of five Iranian EOs and against fluconazole resistant Candida species.

Methods: To determine chemical composition of EOs gas chromatography-mass spectroscopy (GC/MS) was employed. Fluconazole resistant Candida species were chosen and minimum inhibitory concentration (MIC) values of studied EOs were determined by broth microdilution method. Minimum fungicidal concentration (MFC) was determined as the lowest concentration with no fungal growth on solid media. Fungicidal activity was calculated by MFC/MIC ratio. Results: The results showed that C. albicans and C. tropialis isolates were susceptible to itraconazole (ITC) and voriconazole (VRC) while one species of C. glabrata and C. krusei each was resistant to itraconzaole; and itraconazole resistant C. glabrata isolate was resistance to voriconzaole as well. Among tested EOs, the ones from Cinnamomum cayennense, Origanum majorana var. majoranoides and Andropogon citratus had the highest anti-Candida activity. Artemisia aromatica A. Nelson had the highest MIC value against Candida isolates. All EOs in this study had fungicidal activity.

Conclusion: In general, the tested natural compounds are suitable to be used as anti-Candida. However more studies are needed on each chemical compound to evaluate its antifungal activity alone or in combination with other agents.
\end{abstract}

Implication for health policy/practice/research/medical education:

The results of this study revealed that the tested plant essential oils might be used as alternatives to conventional antifungals for treatment of fungal infections.

Please cite this paper as: Nikaein D, Sharifzadeh A, Khosravi AR. Fungicidal versus Fungistatic activity of five Iranian essences against fluconazole resistant Candida species. J Herbmed Pharmacol. 2018;7(4):287-293. doi: 10.15171/jhp.2018.43.

\section{Introduction}

Incidence of invasive fungal infections (IFIs) has been increased in recent years (1). Candida species are the most frequent fungal pathogens isolated from IFIs. Although infections with Candida albicans have been the most prevalent, infections due to non-albicans Candida have been increased significantly in the last decades $(2,3)$. The increase in prevalence of these species might be as a result of higher resistance to conventional antifungals (4).

Development of new antifungals is challenging; because there are only few targets for antifungal actions and toxicity level of many antifungals is relatively high
(5). In recent years, some researchers have focused on natural compounds including essential oils (EOs) and herbal extracts derived from medicinal plants and other biomaterials with antifungal activities (6). EOs are aromatic compounds derived from plant materials (7). EOs have shown to possess antimicrobial, insecticidal and antioxidant properties (8). It is believed that these properties are related to the functions of their components in plants (9). Iran is a country rich in medicinal plants and EOs from these plants could be potent antifungals (10). Conventional antifungals are divided into 2 groups of fungicidal and fungistatic compounds (11). Fungicidal 
activity of antifungals is a benefit in most IFIs, especially in early stages of the disease, including systemic candidiasis, cryptococcal meningitis in HIV patients and aspergillosis in immunocompromised individuals $(12,13)$. This study was designed to evaluate the fungicidal and/or fungistatic activities of 5 Iranian EOs against fluconazole (FLC) resistant Candida species and to study the effect of EOs' chemical compositions on their antifungal properties.

\section{Materials and Methods}

Isolation of Candida species

Candida species were isolated from patients having candidiasis, referred to Mycology Research Center, Faculty of Veterinary Medicine, University of Tehran, Tehran, Iran, during 2016-2018. A total of 50 isolates from different organs were cultured on Sabouraud Dextrose agar (Merck, Germany) at $30^{\circ} \mathrm{C}$ for 7 days. Candida isolates were identified by germ tube test and RAPID yeast plus system (Remel Inc., Lenexa, KS, USA). Isolates were kept at $4^{\circ} \mathrm{C}$ before use (14).

Preparation of essential oils

In the present study, leaves of Artemisia aromatica A. Nelson, Andropogon citratus and Origanum majorana var. majoranoides, bark of Cinnamomum cayennense and flower buds of Syzygium aromaticum were purchased from Pakan Bazr Company, Isfahan, Iran. The plants were authenticated taxonomically at Pharmacognosy Department, Faculty of Pharmacy, University of Tehran, Iran.

EOs were extracted by hydro-distillation of plant parts in a Clevenger-type apparatus at $100^{\circ} \mathrm{C}$ for 5 hours. The EOs were collected and dried over anhydrous sodium sulfate. The chemical composition of each EO was analyzed using a $6890 \mathrm{~N}$ Agilent Gas chromatograph coupled to a 5975 C Agilent Mass-selective detector (Agilent Technologies, Avondale, PA, USA) (15). EOs were stored in a dark glass bottle at $4^{\circ} \mathrm{C}$ until used.

\section{Preparation of antifungal agents}

FLC, itraconazole (ITC) and voriconazole (VRC) standards were prepared from Sigma Company (Sigma, St. Louis, MO, USA). Antifungal stocks were prepared by dissolving $64 \mathrm{mg}$ antifungal in $50 \mathrm{~mL}$ DMSO. Stocks were kept at $-20^{\circ} \mathrm{C}$ before use.

Determination of minimum inhibitory concentration Broth microdilution method was used to study antifungal susceptibility of Candida isolates to mentioned antifungal agents and studied EOs according to Clinical and Laboratory Standards Institute (CLSI) M27-A3 document. In brief, 2 fold serial dilutions of EOs $(15.6,31.2,62.5$, $125,175,250,350,500,700,1000$ and $2000 \mu \mathrm{g} / \mathrm{mL})$ and 3 azole agents $(0.031-64 \mu \mathrm{g} / \mathrm{mL})$ were prepared in 96-well plates using RPMI-1640 media (Sigma, St. Louis, MO,
USA) buffered with MOPS (Sigma, St. Louis, MO, USA). Candida inoculums were adjusted to density of $1 \times 10^{3}$ $5 \times 10^{3} \mathrm{CFU} / \mathrm{mL}$ with the help of a hemocytometer and 0.1 $\mathrm{mL}$ of each inoculum was added to microplate wells which were incubated at $30^{\circ} \mathrm{C}$ for $24-48$ hours. Non-inoculated medium $(200 \mathrm{~mL})$ was considered as negative control (blank), and the well with no EO/azole was spotted as positive control. The growth in each well was compared with the growth in the control well. Minimum inhibitory concentrations (MICs) were visually determined and defined as the lowest concentration of the EO/azole which inhibited growth in comparison with positive control. All tests were done in replicates of three (16).

Determination of minimum fungicidal concentration Wells with no visible growth after 48 hours of incubation were cultured on Sabouraud Dextrose Agar by surface plate count method. Plates were incubated at $30^{\circ} \mathrm{C}$ for 10 days. Minimum fungicidal concentration (MFC) was determined as the lowest concentration with no growth on solid media. All experiments were done with 3 replicates (17).

\section{MFC/MIC ratio}

EOs were considered fungicidal if the ratio of MFC to MIC was less or equal to 4 and if the ratio was higher than four the EO was deemed fungistatic (18).

\section{Statistical analysis}

Data were analyzed using SPSS version 21. Analysis of variance (ANOVA) and Dunnett post hoc test were done to compare different data. A $P$ value of less than 0.05 was considered significant.

\section{Results}

Isolation of Candida species

Specimens were taken from upper respiratory tract, urine and blood. Candida isolates were initially tested for susceptibility to FLC by broth microdilution method. Isolates with MIC range of 64 or greater were considered resistant to FLC. A total of 12 Candida species that were resistant to FLC were selected for further studies. These isolates included C. albicans (6 isolates), Candida glabrata (3 isolates), Candida krusei (2 isolates) and Candida tropicalis (1 isolate).

Analysis of essential oils by Gas chromatography/ Mass spectrometry

EOs were analyzed on a $6890 \mathrm{~N}$ Agilent Gas chromatograph coupled to a 5975 C Agilent mass-selective detector (Agilent Technologies, Avondale, PA, USA) with a 7683 Agilent auto sampler. In brief, $1 \mu \mathrm{L}$ of each sample was injected in the split less mode at $250^{\circ} \mathrm{C} /$ into a $30 \mathrm{~m} \times 0.25$ $\mathrm{mm} \times 0.5 \mu \mathrm{m}$ DB- $5 \mathrm{MS}$ capillary column and operated by MSD Chemstation software (Agilent Technologies).

The temperature program used for the chromatographic 
separation was $50^{\circ} \mathrm{C} / 2 \mathrm{~min} ; 25^{\circ} \mathrm{C} / 1 \mathrm{~min} ; 100^{\circ} \mathrm{C} / 2$ min; $5^{\circ} \mathrm{C} / 1 \mathrm{~min}$ and $290^{\circ} \mathrm{C} / 5 \mathrm{~min}$. The carrier gas was helium (99.999\%) with a constant flux of $1.0 \mathrm{~mL} / \mathrm{min}$. Mass spectrometry was operated in an electron impact ionization mode with the energy of $70 \mathrm{eV}$. Several unknown peaks were observed after injection. Peaks were identified using computer library. The main components of the EOs are summarized in Table 1.

Major compounds of $A$. aromatica A. Nelson were Anethole (22.02\%), d-limonene (14.93\%) and alpha pinene (16.44\%). Isothymol (59.42\%), thymol (15.23\%) and transcaryophyllene $(10.18 \%)$ were the major constituents of A. citratus; while carvacrol (57.86\%), thymol (13.54\%) and trans-caryophyllene $(11.52 \%)$ were the main compounds found in O. majorana var. majoranoides. $C$. cayennense was mainly composed of cinnamaldehyde (62.06\%), cinnamaldehyde dimethyl acetate $(8.14 \%)$ and eugenol (7.49\%). We found eugenol (96.81\%) as the main component of $S$. aromaticum followed by lower percentages of carvacrol (1.74\%) and Trans-Caryophyllene (0.39\%).

\section{Determination of MIC and MFC}

Antifungal susceptibility test of EOs in comparison with ITC and VRC against FLC resistant Candida species was done using broth micro dilution method (Table 2). All C. albicans and C. tropicalis isolates were susceptible to both ITC and VRC. Whereas, isolates of C. krusei and C. glabrata were resistant to ITC. ITC resistant $C$. glabrata was resistant to VRC as well. Among EOs, MICs of $A$. aromatica A. Nelson, A. citratus, O. majorana var. majoranoides, $C$. cayennense and $S$. aromaticum ranged between 1000 to $2000 \mu \mathrm{g} / \mathrm{mL}$ (mean value: $1454.5 \pm$ $509.6 \mu \mathrm{g} / \mathrm{mL}$ ), 125 to $175 \mu \mathrm{g} / \mathrm{mL}$ (mean value: $156.8 \pm$ $24.6 \mu \mathrm{g} / \mathrm{mL}$ ), 173 to $350 \mu \mathrm{g} / \mathrm{mL}$ (mean value: $208 \pm 55.8$ $\mu \mathrm{g} / \mathrm{mL}$ ), 125 to $175 \mu \mathrm{g} / \mathrm{mL}$ (mean value: $147.7 \pm 25.5$ $\mu \mathrm{g} / \mathrm{mL}$ ) and 700 to $1000 \mu \mathrm{g} / \mathrm{mL}$ (mean value: $740.9 \pm$ $105.4 \mu \mathrm{g} / \mathrm{mL}$ ), respectively. O. majorana var. majorana had significantly higher MICs for C. glabrata than other examined Candida species $(P<0.05)$. The MIC ranges of other EOs against Candida species were not statistically significant $(P>0.05)$. C. cayennense could significantly inhibit growth of $C$. albicans in lower concentrations than O. majorana var. majoranoides, $S$. aromaticum and $A$. aromatica A. Nelson $(P<0.05)$. Whilst it was only more effective than $O$. majorana var. majoranoides against $C$. glabrata isolates $(P<0.05)$. The most effective EOs on $C$. krusei isolates were $A$. citratus and $C$. cayennense $(P<0.05)$; and $O$. majorana var. majoranoides was significantly more effective against $C$. tropicalis than $A$. aromatica A. Nelson and $C$. cayennense $(P<0.05)$.

Determination of fungicidal activity

The ration of MIFC/MIC of studied EOs against Candida isolates is shown in Figure 1. Since the MIC/MFC ratios of all EOs in this study were less than 4 , they could be considered fungicidal. C. cayennense MIC/MFC ratio was similar for all Candida species. While A. aromatica A. Nelson had the nearest ratio against C. krusei and other essences had the lowest ratio for C. tropicalis.

\section{Discussion}

Candida species cause a variety of fungal infections from superficial candidiasis of oral and vaginal mucosa to disseminated and deep tissue infections. These infections are most often caused by C. albicans followed by C. glabrata, C. parapsilosis/C. tropicalis and C. krusei according to epidemiological studies in different geographical regions (19). FLC, an azole antifungal, is the most common antifungal used for treatment of candidiasis. It inhibits lanesterol 14 a demethylase enzyme encoded by ERG11 gene. However, some non albicans Candida species have intrinsic resistant to azoles. In addition, Candida spp. are able to develop resistant to azoles $(20,21)$. Azole resistance is seen especially in patients receiving azole prophylaxis (22).

In this study we examined the fungicidal activities of 5 Iranian EOs on FLC resistant to Candida species. Candida yeasts were isolated from candidiasis cases. Resistant to other conventional systemic azoles in Iran was investigated as well. In our study only one isolate of $C$. glabrata was resistant to VRC and one isolate of C. glabrata as well as C. krusei were dose dependently susceptible to ITC. According to recent studies, resistance to azole antifungals has increased in recent years. Widespread use of triazoles has resulted in emergence of resistant to non albicans Candida species including C. glabrata and C. krusei $(23,24)$. These species have intrinsic resistant to FLC and it is said that this inherited resistance could be seen in VRC as well (23). In our study only one isolate of C. glabrata was resistant to VRC. Studies have shown that mutations in ERG11 gene could induce azole resistance in C. albicans (24). Stephanie et al demonstrated that there is a combination effect between FLC and VRC susceptibility in C. albicans strains, while ITC susceptibility is not affected (24). We did not observe any correlation between FLC and VRC susceptibility among our C. albicans isolates. It might be due to different mutation regions in our isolates. Biological activity of EOs is highly related to their chemical compounds (25). In our study, C. cayennense was the most effective one against Candida isolates.

GS/MS analysis revealed cinnamaldehyde, cinnamaldehyde dimethyl acetal and eugenol as its main ingredients. Other studies have demonstrated that the amount of cinnamaldehyde affect antifungal activity of Cinnamomum spp. The higher cinnamaldehyde content the higher antifungal activity would be (26). O. majorana var. majoranoides and A. citratus had the best anti Candida activity following C. cayennense. GC/MS analysis revealed that isothymol and thymol compromised approximately $50 \%$ of A. citratus. These compounds are known for 


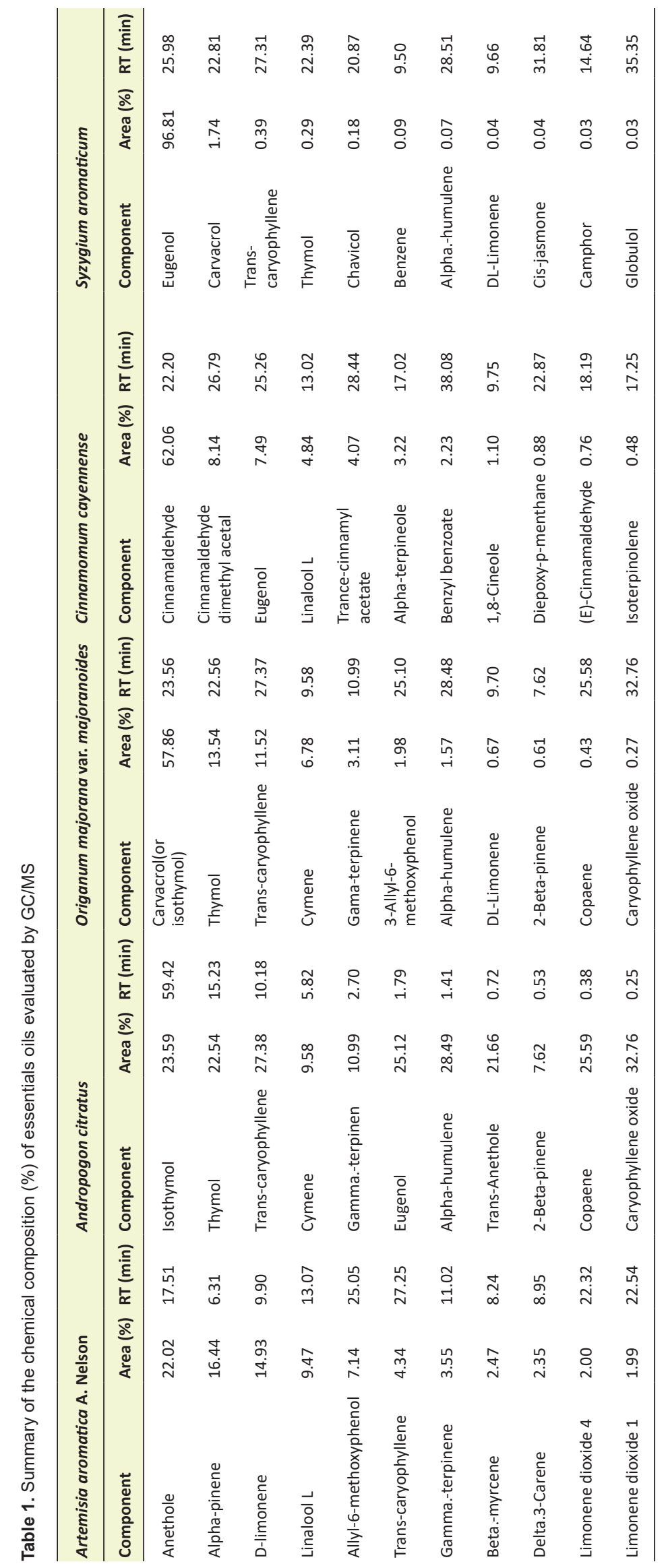


Table 2. Antifungal susceptibility test of selected essential oils in comparison with 3 conventional azole antifungals against Candida isolates

\begin{tabular}{|c|c|c|c|c|c|c|c|c|c|c|c|c|c|c|}
\hline \multirow{2}{*}{$\begin{array}{l}\text { Candida } \\
\text { isolates }\end{array}$} & \multicolumn{2}{|c|}{ Itranconazole } & \multicolumn{2}{|c|}{ Voriconazole } & \multicolumn{2}{|c|}{$\begin{array}{c}\text { Artemisia aromatica } \\
\text { A. Nelson }\end{array}$} & \multicolumn{2}{|c|}{$\begin{array}{l}\text { Andropogon } \\
\text { citratus }\end{array}$} & \multicolumn{2}{|c|}{$\begin{array}{c}\text { Origanum majorana } \\
\text { var. majoranoides }\end{array}$} & \multicolumn{2}{|c|}{$\begin{array}{l}\text { Cinnamomum } \\
\text { cayennense }\end{array}$} & \multicolumn{2}{|c|}{$\begin{array}{l}\text { Syzygium } \\
\text { aromaticum }\end{array}$} \\
\hline & MIC & MFC & MIC & MFC & MIC & MFC & MIC & MFC & MIC & MFC & MIC & MFC & MIC & MFC \\
\hline C. albicans1 & 0.125 & $<0.5$ & 0.125 & $<0.5$ & 2000 & 2000 & 175 & 350 & 175 & 350 & 125 & 175 & 700 & 1000 \\
\hline C. albicans 2 & 0.062 & $<0.5$ & 0.062 & $<0.5$ & 1000 & 2000 & 175 & 350 & 175 & 350 & 125 & 175 & 700 & 1000 \\
\hline C. albicans3 & 0.062 & $<0.5$ & 0.062 & $<0.5$ & 1000 & 2000 & 175 & 350 & 175 & 350 & 125 & 175 & 700 & 1000 \\
\hline C. albicans 4 & 0.062 & $<0.5$ & 0.062 & $<0.5$ & 2000 & 2000 & 175 & 350 & 250 & 350 & 125 & 175 & 700 & 1000 \\
\hline C. albicans5 & 0.062 & $<0.5$ & 0.062 & $<0.5$ & 1000 & 2000 & 125 & 350 & 175 & 350 & 125 & 175 & 700 & 1000 \\
\hline C. albicans 6 & 0.062 & $<0.5$ & 0.062 & $<0.5$ & 1000 & 2000 & 125 & 175 & 175 & 350 & 125 & 175 & 700 & 1000 \\
\hline C. glabrata1 & 4 & $<64$ & 8 & $<64$ & 1000 & 2000 & 175 & 250 & 175 & 250 & 175 & 250 & 700 & 1000 \\
\hline C. glabrata2 & 0.125 & $<0.5$ & 0.25 & $<1$ & 1000 & 2000 & 175 & 250 & 175 & 250 & 175 & 250 & 700 & 700 \\
\hline C. glabrata3 & 0.125 & $<0.5$ & 0.125 & $<0.5$ & 2000 & 2000 & 125 & 175 & 175 & 350 & 175 & 250 & 1000 & 1000 \\
\hline C. krusei1 & 0.062 & $<0.5$ & 0.062 & $<0.5$ & 2000 & 2000 & 125 & 175 & 350 & 500 & 125 & 175 & 1000 & 1000 \\
\hline C. krusei2 & 4 & $<64$ & 0.5 & $<2$ & 1000 & 2000 & 125 & 175 & 350 & 500 & 125 & 175 & 700 & 1000 \\
\hline C. tropicalis & 0.5 & $<2$ & 0.125 & $<0.5$ & 1000 & 2000 & 175 & 175 & 175 & 175 & 175 & 250 & 700 & 700 \\
\hline
\end{tabular}

Data are shown as mean $(\mu \mathrm{g} / \mathrm{mL})$.

their antifungal activities (27). Another compound with high value in A. citratus was Trans-Caryophyllene. It is sesquiterpene found in many EOs especially cloves (28). Trans-Caryophyllene is known for its anti-inflammatory and pain killer effects (29). S. aromaticum activity against FLC resistant Candida species was also suitable ( $\mathrm{MIC}=700$ $\mu / \mathrm{mL}$ ). Only one isolate of C. glabrata and C. krusei had the MIC of $1000 \mu \mathrm{g} / \mathrm{mL}$. Other studies have demonstrated a suitable antifungal activity for eugenol in vivo and in vitro (30). A. aromatica A. Nelson was found to have the lowest antifungal activity against the studied Candida species in our study. Since the numbers of isolates were not similar in our study, we could not predict if the tested EOs act differently on various Candida species, although according to our results there was no difference between FLC resistant Candida species.

All EOs in our study had fungicidal properties. It has been documented that fungicidal activity of antifungals is related to more therapeutic success and less recurrent and persistent infection especially in invasive candidiasis and candidemia $(12,13)$. It has been shown that fungicidal compounds inhibit morphogenic transformation of Candid spp while fungistatics do not affect morphogenesis (18).

\section{Conclusion}

In conclusion, EOs in this study have potential antifungal effects against Candida species and there is no known resistant mechanism in Candida species against studied EOs. These EOs are probably suitable to be used as supplementary agents along with conventional antifungals. However more studies are needed to evaluate their safety on host cells and to examine the effects of their chemical compounds alone and/or in combination with
${ }^{4.0} \quad(\mathrm{~A})$

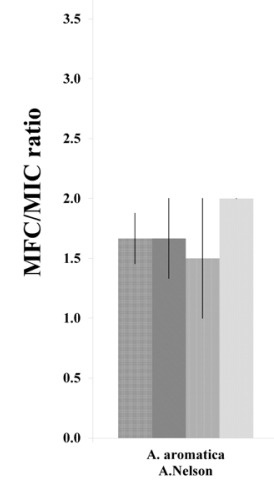

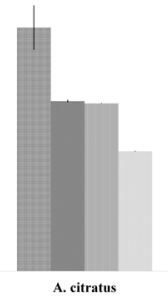
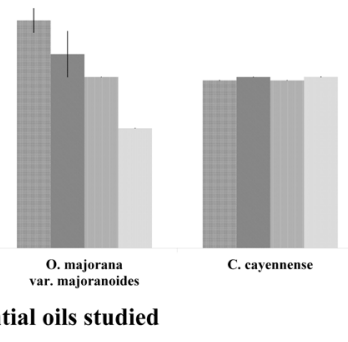

(B)

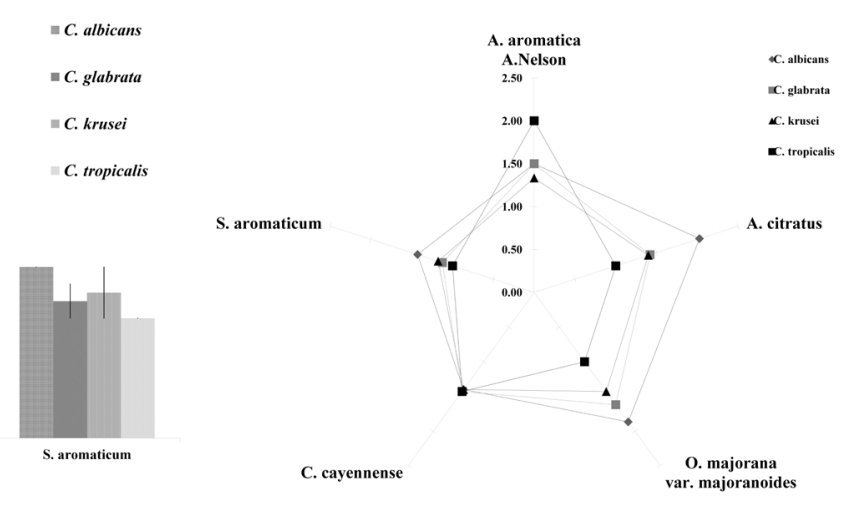

Figure 1. (A) Comparison the MIC/MFC ratio of different essential oils against C. albicans, C. glbrata, C. krusei and C. tropicalis. Data are shown as mean \pm SE. (B) a diagram on relative MIC/MFC ration of studied essential oils. 
other agents.

\section{Authors' contributions}

ARK contributed to study design and manuscript editing. $\mathrm{DN}$ and AS performed experiment and acquired data. DN prepared the manuscript draft. DN submitted the manuscript. All authors read and confirmed final version of the article for publication.

\section{Conflict of interests}

Authors declare no conflict of interest.

\section{Ethical considerations}

This study was approved by Deputy of Research and Technology, Faculty of Veterinary Medicine, University of Tehran (Ethical Code 30792/1/1). All ethical issues (including plagiarism, misconduct, data fabrication, falsification, double publication or submission and redundancy) have been completely observed by the authors.

\section{Funding/Support}

This research was funded and supported by Research Council of University of Tehran (Grant No. 30792/1/1)

\section{References}

1. Brown GD, Denning DW, Levitz SM. Tackling human fungal infections. Science. 2012;336(6082):647. doi: 10.1126/science.1222236.

2. Falagas ME, Roussos N, Vardakas KZ. Relative frequency of albicans and the various non-albicans Candida spp among candidemia isolates from inpatients in various parts of the world: a systematic review. Int J Infect Dis. 2010;14(11):e954-66. doi: 10.1016/j.ijid.2010.04.006.

3. Oberoi JK, Wattal C, Goel N, Raveendran R, Datta S, Prasad K. Non-albicans Candida species in blood stream infections in a tertiary care hospital at New Delhi, India. Indian J Med Res.2012;136(6):997-1003.

4. Pfaller MA, Diekema DJ, Gibbs DL, Newell VA, Ellis D, Tullio V, et al. Results from the ARTEMIS DISK Global Antifungal Surveillance Study, 1997 to 2007: a 10.5-year analysis of susceptibilities of Candida species to fluconazole and voriconazole as determined by CLSI standardized disk diffusion. J Clin Microbiol. 2010;48(4):1366-77. doi: 10.1128/jcm.02117-09.

5. Perfect JR. The antifungal pipeline: a reality check. Nat Rev Drug Discov. 2017;16(9):603-16. doi: 10.1038/nrd.2017.46.

6. Raimundo KF, Bortolucci WC, Glamoclija J, Sokovic M, Goncalves JE, Linde GA, et al. Antifungal activity of Gallesia integrifolia fruit essential oil. Braz J Microbiol. 2018. doi: 10.1016/j.bjm.2018.03.006.

7. Hussain AI, Anwar F, Hussain Sherazi ST, Przybylski R. Chemical composition, antioxidant and antimicrobial activities of basil (Ocimum basilicum) essential oils depends on seasonal variations. Food Chem. 2008;108(3):986-95. doi: 10.1016/j.foodchem.2007.12.010.

8. Chis MS, Muste S, Paucean A, Man S, Sturza A, Petrut GS, et al. A comprehensive review about antimicrobial effects of herb and oil oregano (Origanum vulgare ssp. Hirtum). Hop Med Plants. 2017;25(1-2):17-27.

9. Mahmoud SS, Croteau RB. Strategies for transgenic manipulation of monoterpene biosynthesis in plants. Trends Plant Sci. 2002;7(8):366-73.

10. Bazdar M, Sadeghi H, Hosseini S. Evaluation of oil profiles, total phenols and phenolic compounds in Prangos ferulacea leaves and flowers and their effects on antioxidant activities. Biocatal Agric Biotechnol. 2018;14:418-23. doi: 10.1016/j. bcab.2018.04.009.

11. Lewis JS 2nd, Graybill JR. Fungicidal versus Fungistatic: what's in a word? Expert Opin Pharmacother. 2008;9(6):92735. doi: 10.1517/14656566.9.6.927.

12. Reboli AC, Rotstein C, Pappas PG, Chapman SW, Kett $\mathrm{DH}$, Kumar D, et al. Anidulafungin versus fluconazole for invasive candidiasis. N Engl J Med. 2007;356(24):2472-82. doi: 10.1056/NEJMoa066906.

13. Cornely OA, Maertens J, Bresnik M, Ebrahimi R, Ullmann AJ, Bouza E, et al. Liposomal amphotericin B as initial therapy for invasive mold infection: a randomized trial comparing a high-loading dose regimen with standard dosing (AmBiLoad trial). Clin Infect Dis. 2007;44(10):128997. doi: $10.1086 / 514341$.

14. Darwazeh AM, Al-Refai S, Al-Mojaiwel S. Isolation of Candida species from the oral cavity and fingertips of complete denture wearers. J Prosthet Dent. 2001;86(4):4203. doi: $10.1067 / \mathrm{mpr} .2001 .118020$.

15. Adams RP. Identification of essential oil components by gas chromatography/mass spectrometry. Carol Stream, IL: Allured Publishing Corporation; 2007.

16. Espinel-Ingroff A, Canton E, Peman J, Rinaldi MG, Fothergill AW. Comparison of 24-hour and 48-hour voriconazole MICs as determined by the Clinical and Laboratory Standards Institute broth microdilution method (M27-A3 document) in three laboratories: results obtained with 2,162 clinical isolates of Candida spp. and other yeasts. J Clin Microbiol. 2009;47(9):2766-71. doi: 10.1128/ jcm.00654-09.

17. Mann A, Banso A, Clifford LC. An antifungal property of crude plant extracts from Anogeissus leiocarpus and Terminalia avicennioides. Tanzan J Health Res. 2008;10(1):34-8.

18. Hawser S, Islam K. Comparisons of the effects of fungicidal and fungistatic antifungal agents on the morphogenetic transformation of Candida albicans. J Antimicrob Chemother. 1999;43(3):411-3.

19. Whaley SG, Berkow EL, Rybak JM, Nishimoto AT, Barker KS, Rogers PD. Azole antifungal resistance in Candida albicans and emerging non-albicans Candida species. Front Microbiol. 2016;7:2173. doi: 10.3389/ fmicb.2016.02173.

20. Sharifzadeh A, Shokri H. Antifungal activity of essential oils from Iranian plants against fluconazole-resistant and fluconazole-susceptible Candida albicans. Avicenna J Phytomed. 2016;6(2):215-22.

21. Lortholary O, Renaudat C, Sitbon K, Madec Y, DenoeudNdam L, Wolff M, et al. Worrisome trends in incidence and mortality of candidemia in intensive care units (Paris area, 2002-2010). Intensive Care Med. 2014;40(9):1303-12. doi: 10.1007/s00134-014-3408-3. 
22. Hachem R, Hanna H, Kontoyiannis D, Jiang Y, Raad I. The changing epidemiology of invasive candidiasis: Candida glabrata and Candida krusei as the leading causes of candidemia in hematologic malignancy. Cancer. 2008;112(11):2493-9. doi: 10.1002/cncr.23466.

23. Perlin DS, Shor E, Zhao Y. Update on antifungal drug resistance. Curr Clin Microbiol Rep. 2015;2(2):84-95. doi: 10.1007/s40588-015-0015-1.

24. Flowers SA, Colon B, Whaley SG, Schuler MA, Rogers PD. Contribution of clinically derived mutations in ERG11 to azole resistance in Candida albicans. Antimicrob Agents Chemother. 2015;59(1):450-60. doi: 10.1128/aac.03470-14.

25. Radu N, Voicescu M, Radu E, Tanasescu C. Biomaterial with antioxidant and antifungal activities, obtained from Romanian indigenous plants. Molecular Crystals and Liquid Crystals. 2017;655(1):243-9. doi: $10.1080 / 15421406.2017 .1361732$.

26. Cheng SS, Liu JY, Hsui YR, Chang ST. Chemical polymorphism and antifungal activity of essential oils from leaves of different provenances of indigenous cinnamon
(Cinnamomum osmophloeum). Bioresour Technol. 2006;97(2):306-12. doi: 10.1016/j.biortech.2005.02.030.

27. Shu C, Sun L, Zhang W. Thymol has antifungal activity against Candida albicans during infection and maintains the innate immune response required for function of the p38 MAPK signaling pathway in Caenorhabditis elegans. Immunol Res. 2016;64(4):1013-24. doi: 10.1007/s12026016-8785-y.

28. Tongnuanchan P, Benjakul S. Essential oils: extraction, bioactivities, and their uses for food preservation. J Food Sci. 2014;79(7):R1231-49. doi: 10.1111/1750-3841.12492.

29. Paula-Freire LI, Andersen ML, Gama VS, Molska GR, Carlini EL. The oral administration of trans-caryophyllene attenuates acute and chronic pain in mice. Phytomedicine. 2014;21(3):356-62. doi: 10.1016/j.phymed.2013.08.006.

30. Rana IS, Rana AS, Rajak RC. Evaluation of antifungal activity in essential oil of the Syzygium aromaticum (L.) by extraction, purification and analysis of its main component eugenol. Braz J Microbiol. 2011;42(4):1269-77. doi: 10.1590/s1517-83822011000400004. 